\title{
Radio Controlled “3D Aerobatic Airplanes" as Basis for Fixed-Wing UAVs with VTOL Capability
}

\author{
Chung-How Poh, Chung-Kiak Poh \\ Aero-Persistence Research, Halaman York, Malaysia \\ Email: Chung-How@ieee.org
}

Received 30 September 2014; revised 31 October 2014; accepted 5 November 2014

Academic Editor: Frédéric Muttin, EIGSI Ecole d'Ingénieurs La Rochelle, La Rochelle Area, France

Copyright (C) 2014 by authors and Scientific Research Publishing Inc.

This work is licensed under the Creative Commons Attribution International License (CC BY).

http://creativecommons.org/licenses/by/4.0/

(c) $\underset{\mathrm{EY}}{\mathrm{B}}$ Open Access

\begin{abstract}
There are fundamental performance compromises between rotary-wing and fixed-wing UAVs. The general solution to address this well-known problem is the design of a platform with some degree of reconfigurable airframes. For critical missions (civilian or military), it is imperative that mechanical complexity is kept to a minimum to help achieve mission success. This work proposes that the tried-and-true radio controlled (RC) aerobatic airplanes can be implemented as basis for fixed-wing UAVs having both speed and vertical takeoff and landing (VTOL) capabilities. These powerful and highly maneuverable airplanes have non-rotatable nacelles, yet capable of deep stall maneuvers. The power requirements for VTOL and level flight of an aerobatic RC airplane are evaluated and they are compared to those of a RC helicopter of similar flying weight. This work provides quantitative validation that commercially available RC aerobatic airplanes can serve as platform to build VTOL capable fixed-wing UAVs that are agile, cost effective, reliable and easy maintenance.
\end{abstract}

\section{Keywords}

Aerobatics, Unmanned Aerial Vehicle, Fixed-Wing, VTOL, Hover

\section{Introduction}

Unmanned aerial vehicles (UAVs) are being recognized as cost-effective alternative to manned-aircraft in carrying out various civilian and military missions [1]-[5]. Among the important civilian applications of UAV are 
natural disaster prevention and management [6]-[8], as well as emergency response and aid delivery [9]-[12]. General statement regarding the choice of UAV platform is as follows: rotary-wing UAVs have the ability to hover, take off and land vertically and with agile maneuvering capability at the expense of high mechanical complexity, low speed and short flight range. On the other hand, fixed-wing UAVs are able to cruise efficiently at high speeds for longer duration though runway is required for take-off and landing [13]-[15]. The innovation of the tiltrotor was to take advantage of the VTOL capability of a rotor-wing and the speed and range of a conventional fixed-wing aircraft. It has a rotating engine nacelles to direct thrust but the aircraft design was vulnerable to dangerous aerodynamic phenomena and reliability issues [16] [17].

Any radio controlled (RC) airframe can potentially be used to create an UAV, whether it is in the form of a fixed-wing or a rotary-wing aircraft. An UAV generally has a higher level of sophistication in its navigation and auto-pilot system. Modern aerobatic fixed-wing aircraft, whether full-size or not, or RC models were designed to operate even under fully stalled conditions [18]-[23]. In particular, these fixed-wing aircraft can fly in trim at high angles of attack near $45^{\circ}$ even with flight speed below the stall speed, a maneuver known as the "harrier" which is one of the popular "3D aerobatic maneuvers" within the RC flight community. Other 3D aerobatics that have been routinely performed by RC pilots are the hovering, flat-spin, blender, waterfall, tailslide and their derivatives [18] [20] [23]. Hallmarks of unlimited aerobatic airplanes are their relatively large control surfaces immersed in strong propeller wash and thrust-to-weight ratio that exceeds 1 [18] [19].

Large control surfaces with large deflections in the presence of strong propeller wash give adequate authority even when the airspeed of the aircraft is much lower than the stall speed $\left(V_{s}\right)$. This enables an aerobatic model airplane to be maneuverable in the deep wing-stall condition. Many fixed-wing UAVs have been designed with relatively small control surfaces [24] especially those that have been inspired by the military UAVs such as the MQ-9 Reaper by General Atomics. Small control surfaces exert much weaker force even in strong propeller wash and this limits the ability of the airplane to make corrective input during hovering despite having an engine that can produce powerful thrust. Figure 1 shows two RC fixed-wing airplanes belonging to the authors. The Cirrus SR-22 represented the more conventional airframe while the Katana-50 has features shared in common with any typical 3D aerobatic airplanes. Note that the percents of chord at tip and root of the large elevator on the Katana-50 were approximately 50\%, in contrast to those of the Cirrus SR-22. This allows the elevator to continue having control over the pitch of the aircraft during the vertical hovering maneuver. Likewise, its large rudder and ailerons have authoritative control over the yaw and roll of the aircraft during hovering and other post wing-stall maneuvers.

This study employed a commercially available 25\% scale RC Extra 260 (electric-powered) as the agile platform and validates quantitatively that hovering and VTOL maneuvers are well within the flight envelope of

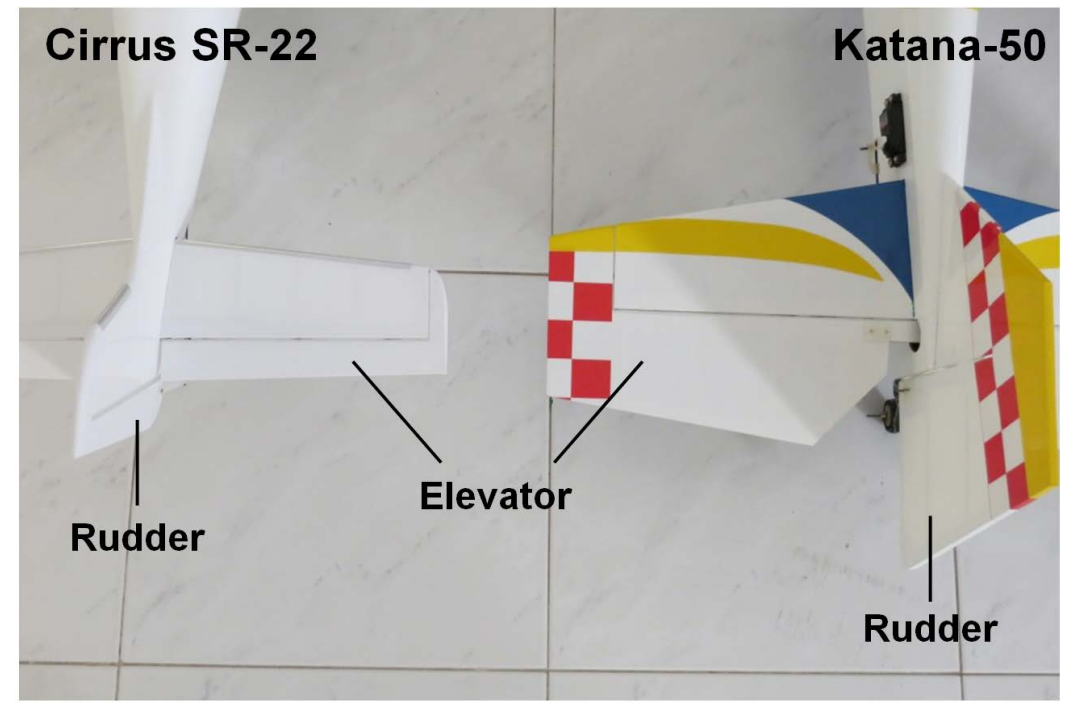

Figure 1. Comparison of flight control surfaces (rudder and elevator) on the Cirrus SR-22 and the Katana-50. Large control surfaces allow 3D aerobatic airplanes to easily perform post wing-stall maneuvers such as the "harrier". 
fixed-wing UAV without resorting to configuration involving rotating nacelles while retaining common advantages shared with conventional fixed-wing aircraft such as efficient cruising. The motivation for this work is that if such simple yet robust platform proves to be a viable option, then the approach will expedite the development and deployment of VTOL-capable fixed-wing UAVs without the formidable costs and mechanical complexities. Low cost and ease of mass production of such platform will indirectly promote the practical implementation of large-scale multi-agent UAVs with unique emergent collective behaviors. Power consumptions at different phases of flight are investigated in this work via experimental field testing.

What is related to this research work is the recently revealed Project Wing by Google X [25]. The advantage of 3D airplane proposed in this work is the maturity of its production process. Furthermore, the flight performance, robustness and reliability of such airframes have put to test in international competitions such as the prestigious Fédération Aéronautique Internationale (FAI) Class F3M. They also have been indirectly tested by RC pilots around the world during their weekend flying sessions since the concept was conceived along with the introduction of lithium batteries for RC applications about 10 years ago. These resulted in tremendous accumulated hours of rigorous testing and technical know-how.

\section{Materials and Methods}

A 3D aerobatic airplane was used in this study and a pod-and-boom helicopter was included as comparison. Both of them were electric powered. The airplane was a 25\% scale Extra 260 from Hobby King [26] and the helicopter was a Logo-10 by Mikado Model Helicopters [27] with custom stretched tail-boom to accept $600 \mathrm{~mm}$ main rotor blades. The Extra 260 and the stretched Logo-10 are as shown in Figure 2(a) and Figure 2(b), respectively. Both the wing and the rotor blades have symmetrical airfoils. Maximum deflections of ailerons, elevator, and rudder were $40^{\circ}, 40^{\circ}$ and $45^{\circ}$, respectively for the Extra 260 . Setup details of the models were tabulated in Table 1. Chemistry of batteries used for all the model aircraft was lithium polymer (Li-po). Additional mass was added to the Logo-10 so that both aircraft have identical flying weight of $4.5 \mathrm{~kg}$. $5.8 \mathrm{GHz}$ telemetry system by Guilin Feiyu Electronic Technology Co., Ltd. was employed in this study to relay real time data for

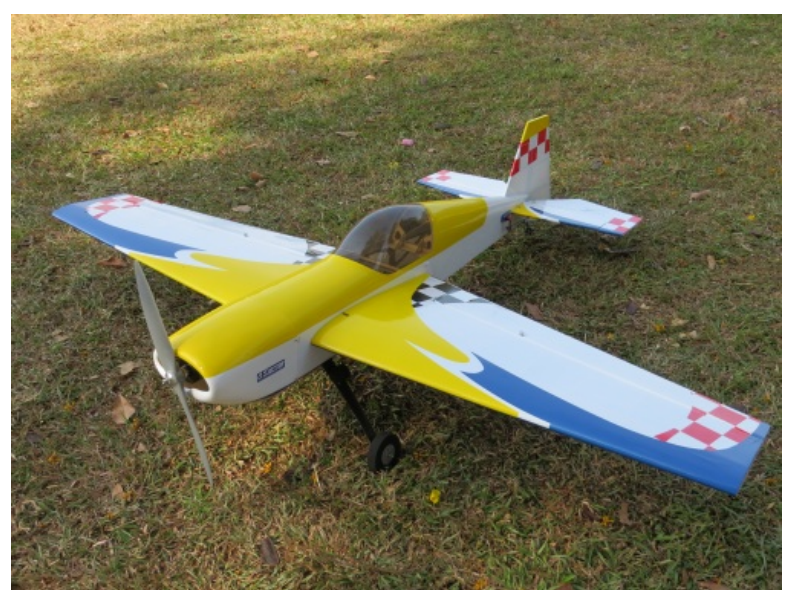

(a)

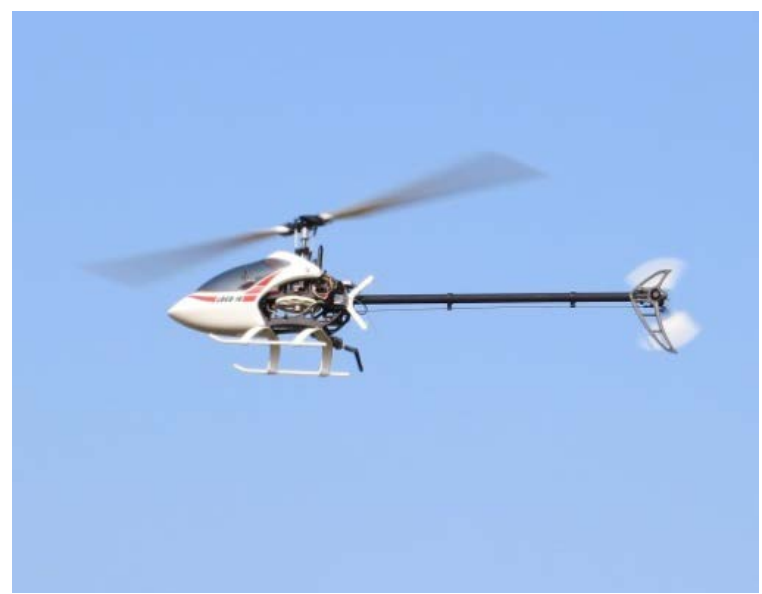

(b)

Figure 2. RC model aircraft employed in this study: (a) 25\% scale Extra 260; (b) Logo-10 with stretched tail-boom to accept $600 \mathrm{~mm}$ main blades.

Table 1. Key setup of the model aircraft.

\begin{tabular}{|c|c|c|}
\hline & $25 \%$ scale Extra 260 & Logo-10 \\
\hline Manufacturer & Hobby King & Mikado Model Helicopters \\
\hline Airframe type & Fixed-wing & Rotary-wing \\
\hline Wing span/main rotor diameter (mm) & 1754 & 1340 \\
\hline Wing/disk loading $\left(\mathrm{g} \cdot \mathrm{dm}^{-2}\right)$ & 71.8 & 31.3 \\
\hline Total flying weight (kg) & 4.50 & 4.50 \\
\hline
\end{tabular}


monitoring and the data were stored on a nonvolatile memory card for subsequent analysis. The primary sub-components of the telemetry system were the GPS sensor, overlay screen display (OSD) unit, and current sensor. The current sensor chip was the ACS758LCB-100B by Allegro Microsystems and was pre-calibrated at factory up to $100 \mathrm{~A}$ and the unit was also capable of measuring the input voltage to the electric motor. Electrical power was obtained by multiplying the current and voltage developed across the motors. The flying weight of the models was gauged using a digital weighing scale with a resolution of $10 \mathrm{~g}$. Experimental flights were performed in calm air (wind speed $<5 \mathrm{~km} \cdot \mathrm{h}^{-1}$ ) and "straight and level flight" was maintained with near-zero vertical speed during data acquisition. Radio transmitter used for the flight control was the Futaba 10CG.

\section{Results and Discussion}

Hovering and VTOL capabilities were clearly demonstrated for the Extra 260 and these attributes were typical of any RC 3D airplanes with thrust/weight ratio that exceeds unity. It achieved a vertical climb rate of about 5 $\mathrm{m} \cdot \mathrm{s}^{-1}$ and it also demonstrated a maximum stable descent rate of $3 \mathrm{~m} \cdot \mathrm{s}^{-1}$ without any adverse characteristics. These vertical rates enabled the airplane to perform VTOL with good margin of safety. Moderate deflection of the ailerons (not exceeding $18^{\circ}$ ) was adequate to counteract the propeller torque effect during the VTOL and the "harrier" maneuver. Concept of co-axial counter-rotating propellers could be implemented to reduce the propeller torque effect and the P-factor to an almost negligible level. This would, to a large extend, eliminate the need to apply ailerons during harrier or VTOL. Counter-rotating propeller systems, such as the contra-drive system by SebArt-Hacker, are currently available for high-end competition F3A models [28].

The electrical power consumption at steady level flight and in trim at high angles of attack for the Extra 260 was plotted in Figure 3. The flight power for Logo-10 was included for comparison. The Extra 260's power curve was nonlinear and displayed a minimum. The point on the curve tangent to a horizontal line is the minimum power value, and the corresponding airspeed is the minimum power airspeed $\left(V_{m p}\right)$. A line drawn from the origin and tangent to the curve will produce the airspeed of minimum drag $\left(V_{m d}\right)$ at the point of tangent. Power curve for the Extra 260 also exhibited the well-known "Region of Normal Command" and "Region of Reverse Command" [29]. As evidenced from Figure 3, a unique characteristic of such platform was that the large control surfaces continued to have authority even in the "Region of Reverse Command" down to zero airspeed (vertical hovering). This very characteristic enabled the full retention of control over the roll, pitch, and yaw axes. At the same time, the thrust-to-weight ratio of 1.3 was sufficient to keep it airborne in the deep wing-stall regime. From Figure 3, the $V_{m p}$ and $V_{m d}$ of the Extra 260 were $40 \mathrm{~km} \cdot \mathrm{h}^{-1}$ and $55 \mathrm{~km} \cdot \mathrm{h}^{-1}$, respectively, and the ratio of $V_{m p} / V_{m d}$ was in good agreement with the theoretical value of $3^{-1 / 4}$ neglecting compressibility drag.

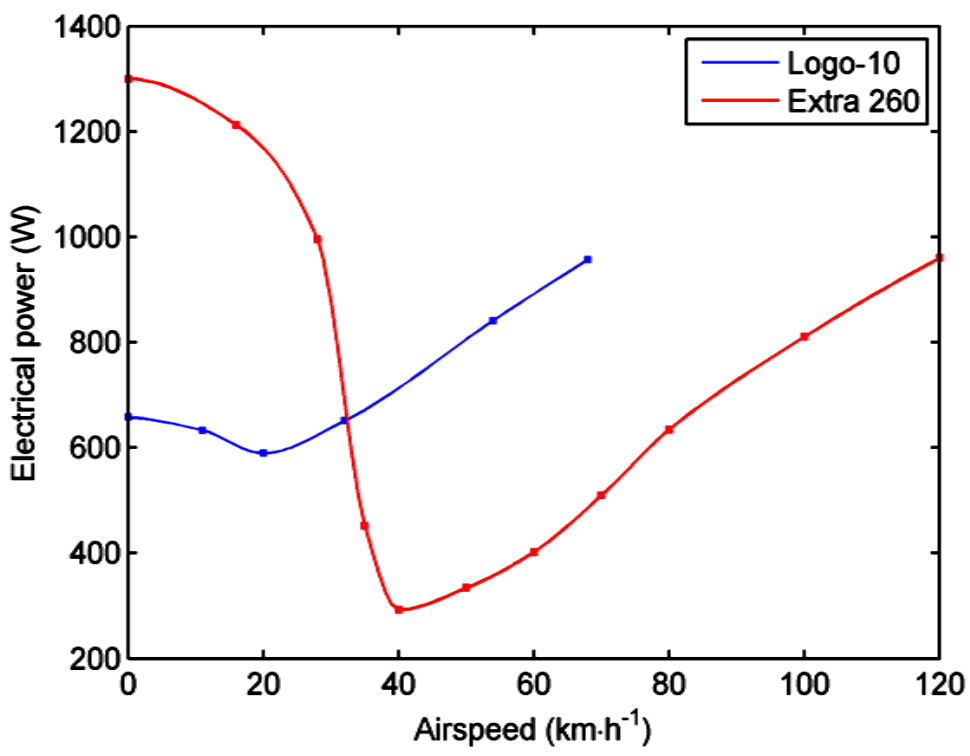

Figure 3. Power requirement for the UAV rotorcraft (Logo-10) and the 3D fixed-wing aerobatic aircraft (Extra 260). The ratio of $V_{m p} / V_{m d}$ for the Extra 260 is $72.7 \%$. Flying weights of both aircraft were $4.5 \mathrm{~kg}$. 
Results in Figure 3 supported the well-known fact that fixed-wing aircraft can cruise much more efficiently compared with rotorcrafts of similar flying weight and their ability to cover greater distance at higher speed. The Extra 260 achieved a $V_{h}$ (maximum speed in level flight with maximum continuous power) of $120 \mathrm{~km} \cdot \mathrm{h}^{-1}$ with a corresponding power of $960 \mathrm{~W}$. As mentioned, a common disadvantage associated with fixed-wing aircraft is the need of runways for taking off and landing. However, Figure 3 provided the quantitative evidence that breaks the stigma and validated that 3D fixed-wing aerobatic aircraft can indeed hover and perform VTOL without involving complex movable mechanical components. Mitigation of complex mechanism would result in greater reliability required for mission success and increased operational safety. As the Extra 260's power requirements in the slow flight deep stall phase were about 3 times more than those at $V_{m p}$ or $V_{m d}$, it is recommended that VTOL, hovering and "harrier" maneuvers should only be performed when necessary to conserve onboard energy storage for optimum endurance.

The Logo-10 was able to hover more efficiently than the Extra 260 (658 W vs. $1300 \mathrm{~W}$ ) mainly because its main rotor was significantly larger than the propeller of the Extra 260. As the Logo-10 made the transition to forward flights, there was a slight drop in the power requirement, reaching a minimum around $20 \mathrm{~km} \cdot \mathrm{h}^{-1}$ because of the translational lift effect. However, at $35 \mathrm{~km} \cdot \mathrm{h}^{-1}$ and above, its power requirement was significantly higher than that of the Extra 260. While the highest airspeed data collected for the Logo-10 was $78 \mathrm{~km} \cdot \mathrm{h}^{-1}$, it was nevertheless sufficient to provide general comparison of flight power requirement between fixed-wing and rotary-wing aircraft. Figure 4(a) and Figure 4(b) are the optical images captured by the rearward facing camera attached onto the landing gear of the Extra 260 during the cruising and VTOL phase, respectively. Snapshot in Figure 4(b) shows the edge of the flying field with some vegetation and an asphalt road. Mature manufacturing techniques, simple and robust airframes of these 3D aerobatic platforms will help encourage the growth of cost-effective multi-agent fixed-wing UAVs with smart cooperative behaviors which undoubtedly will find important applications such as in large-scale search and rescue operation and rapid mapping of a hilly region in the aftermath of a massive landslide.

\section{Conclusion}

This work provided quantitative validation that commercially available unlimited aerobatic RC airplanes can serve as basis for VTOL-capable fixed-wing UAVs with simple airframe and avoid complex mechanical components associated with reconfigurable wings. This attribute will translate into low manufacturing cost, ease of maintenance and significantly higher rate of mission success. Experimental results based on a commercially available 25\% scale Extra 260 indicated that the concept is indeed viable and such platform has significantly broader trimmable flight envelope compared with the more conventional fixed-wing aircraft with smaller control surfaces. It has demonstrated ability to perform impressive post wing-stall maneuvers such as high-angle slow flight ("harrier") and hovering. Low cost and reliability aspects of such agile platform can help expedite the realization of large-scale multi-agent fixed-wing UAV systems with emergent collective behaviors.

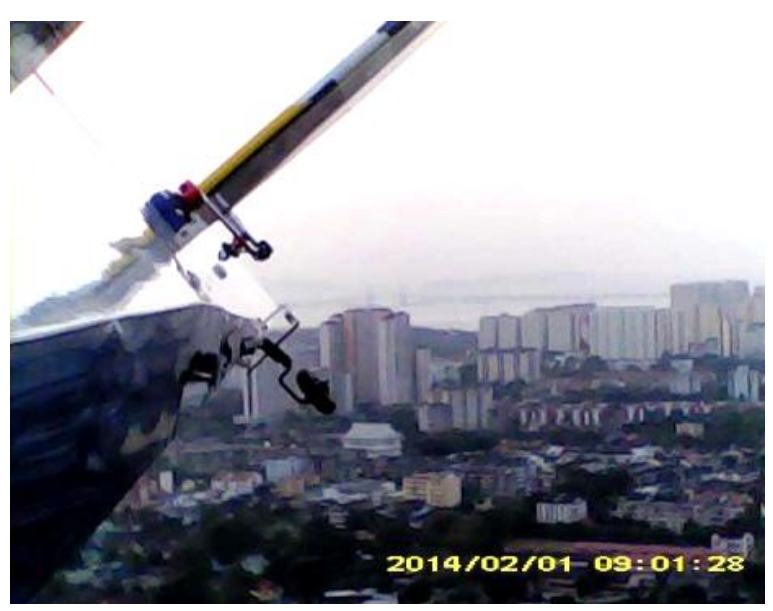

(a)

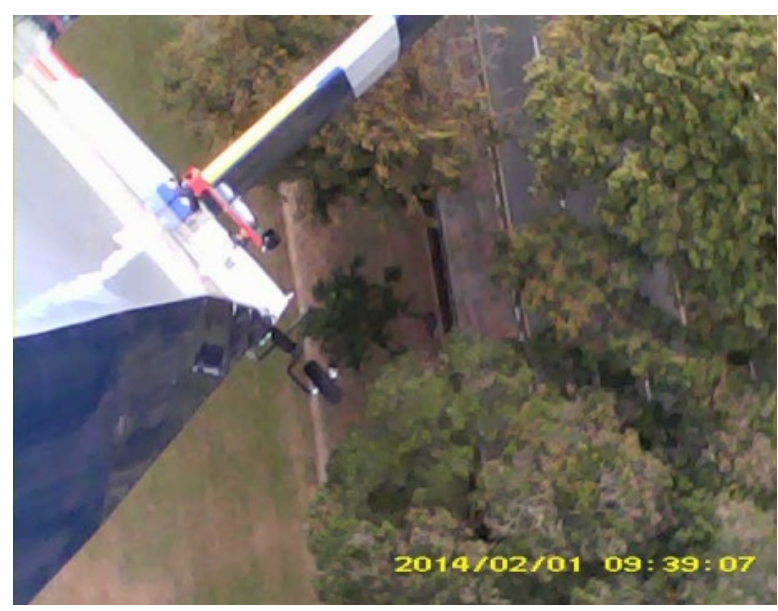

(b)

Figure 4. Image taken by the Extra 260's rearward facing camera during (a) cruising and (b) VTOL. 


\section{References}

[1] Mulero-Pázmány, M., Negro, J.J. and Ferrer, M. (2014) A Low Cost Way for Assessing Bird Risk Hazards in Power Lines: Fixed-Wing Small Unmanned Aircraft Systems. Journal of Unmanned Vehicle Systems, 2, 5-15. http://dx.doi.org/10.1139/juvs-2013-0012

[2] Capata, R., Marino, L. and Sciubba, E. (2014) A Hybrid Propulsion System for a High-Endurance UAV: Configuration Selection, Aerodynamic Study, and Gas Turbine Bench Tests. Journal of Unmanned Vehicle Systems, 2, 16-35. http://dx.doi.org/10.1139/juvs-2013-0005

[3] Fahlstrom, P. and Gleason, T. (2012) Introduction to UAV Systems. 4th Edition, John Wiley \& Sons, West Sussex, 1-31. http://dx.doi.org/10.1002/9781118396780.part1

[4] Koh, L.P. and Wich, S.A. (2012) Dawn of Drone Ecology: Low-Cost Autonomous Aerial Vehicles for Conservation. Tropical Conservation Science, 5, 121-132.

[5] Austin, R. (2010) Unmanned Aircraft Systems. John Wiley \& Sons, West Sussex, 1-15. http://dx.doi.org/10.1002/9780470664797.ch1

[6] Maza, I., Caballero, F., Capitán, J., Martínez-de-Dios, J.R. and Ollero, A. (2011) Experimental Results in Multi-UAV Coordination for Disaster Management and Civil Security Applications. Journal of Intelligent and Robotic Systems, 61, 563-585. http://dx.doi.org/10.1007/s10846-010-9497-5

[7] Niethammer, U., James, M.R., Rothmund, S., Travelletti, J. and Joswig, M. (2012) UAV-Based Remote Sensing of the Super-SauzeLandslide: Evaluation and Results. Engineering Geology, 128, 2-11.

[8] Ackerman, E. (2011) Japan Earthquake: Global Hawk UAV May Be Able to Peek inside Damaged Reactors. IEEE Spectrum.

http://spectrum.ieee.org/automaton/robotics/military-robots/global-hawk-uav-may-be-able-to-peek-inside-damaged-rea ctors

[9] Ackerman, E. (2013) Drone Adventures Uses UAVs to Help Make the World a Better Place. IEEE Spectrum. http://spectrum.ieee.org/automaton/robotics/aerial-robots/drone-adventures-uses-uavs-to-help-people-make-the-worlda-better-place

[10] Andrade, R. (2013) Drones Begin to Show Their Development Promise. http://www.scidev.net/global/biodiversity/feature/drones-begin-to-show-their-development-promise.html

[11] Melville, N.A. (2013) Drones, Smartphones Eyed for Medical Aid to Remote Regions. http://www.medscape.com/viewarticle/811951

[12] Hanlon, M. (2013) Hirobo Unveils HX-1 Unmanned Electric Helicopter, and Promises a Manned Model. http://www.gizmag.com/hirobo-personal-manned-micro-electric-helicopter/29684/

[13] Ünel, M., Gülez, K., Çetinsoy, E., Sırımoğlu, E., Öner, K.T., Hançer, C., Akşit, M.F. and Kandemir, İ. (2011) Design and Development of a Tilt-Wing UAV. Turkish Journal of Electrical Engineering \& Computer Sciences, 19, 733-741.

[14] Carlson, E. and Zhao, Y. (2004) Optimal City-Center Takeoff Operation of Tiltrotor Aircraft in One Engine Failure. Journal of Aerospace Engineering, 17, 26-39. http://dx.doi.org/10.1061/(ASCE)0893-1321(2004)17:1(26)

[15] Ozdemir, U., Aktas, Y.O., Vuruskan, A., Dereli, Y., Tarhan, A.F., Demirbag, K., Erdem, A., Kalaycioglu, G.D., Ozkol, I. and Inalhan, G. (2014) Design of a Commercial Hybrid VTOL UAV System. Journal of Intelligent \& Robotic Systems, 74, 371-393. http://dx.doi.org/10.1007/s10846-013-9900-0

[16] Axe, D. (2011) Osprey Down: Marines Shift Story on Controversial Warplane’s Safety Record. http://www.wired.com/2011/10/osprey-down/all/1

[17] Axe, D. (2011) “Son of Osprey” Could Replace All Army Copters. http://www.wired.com/2011/10/son-of-osprey/

[18] International Miniature Aerobatic Club (2009) About IMAC. http://www.mini-iac.com/

[19] Selig, M.S. (2010) Modeling Full-Envelope Aerodynamics of Small UAVs in Real Time. Proceedings of the AIAA Atmospheric Flight Mechanics Conference, Toronto, 2-5 August 2010, 7635.

[20] Academy of Model Aeronautics (2013) Radio Control Scale Aerobatics. http://www.modelaircraft.org/files/2013-2014RCScaleAerobatics1-1.pdf

[21] Zivko Aeronautics Inc. (2005) Edge Aircraft. http://www.zivko.com/edge/site.html

[22] Extra Aircraft (2014) Extra Aerobatic Planes. http://www.extraaircraft.com/

[23] Fédération Aéronautique Internationale (2014) Powered Aerobatics. http://www.fai.org/civa-our-sport/powered-aerobatics

[24] Watts, A.C., Ambrosia, V.G. and Hinkley, E.A. (2012) Unmanned Aircraft Systems in Remote Sensing and Scientific Research: Classification and Considerations of Use. Remote Sensing, 4, 1671-1692. 
http://dx.doi.org/10.3390/rs4061671

[25] Stewart, J. (2014) Google Tests Drone Deliveries in Project Wing Trials. http://www.bbc.com/news/technology-28964260

[26] Hobby King (2013) Extra 260 3D 1754mm 26cc 30cc Gas (ARF). http://www.hobbyking.com/hobbyking/store/_19798_Extra_260_3D_1754mm_26cc_30cc_Gas_ARF_US_Warehou se_html

[27] Mikado Model Helicopters (2014) Logo-10.

http://shop.mikado-heli.de/Weitere-LOGOs/LOGO-10.htm?shop=k_mikado\&SessionId=\&a=catalog\&t=4681\&c=256 $3 \& p=2563$

[28] Ramel, M. (2013) Coaxial Drive Propulsion for F3A Models.

http://www.sebart.it/img-F3A/coaxial-drive/coaxial-drive-description.html

[29] Cavcar, M. (2004) Minimum Drag Speed. http://home.anadolu.edu.tr/ mcavcar/htk224/MinDrag.pdf 
Scientific Research Publishing (SCIRP) is one of the largest Open Access journal publishers. It is currently publishing more than 200 open access, online, peer-reviewed journals covering a wide range of academic disciplines. SCIRP serves the worldwide academic communities and contributes to the progress and application of science with its publication.

Other selected journals from SCIRP are listed as below. Submit your manuscript to us via either submit@scirp.org or Online Submission Portal.
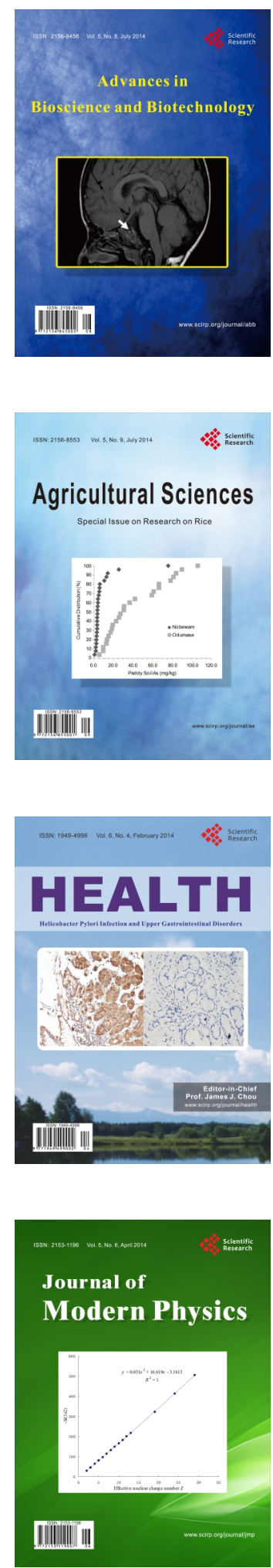
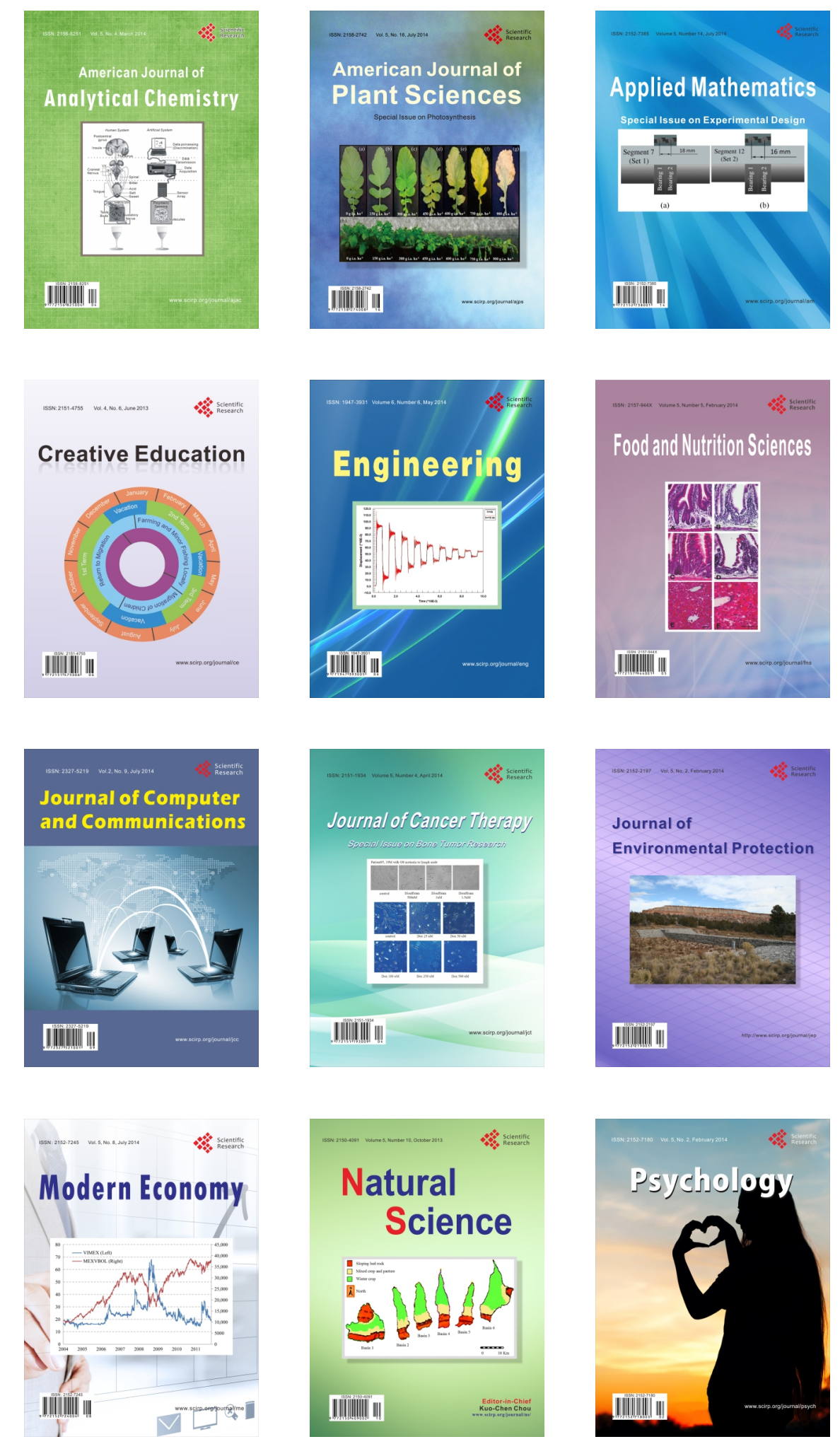